\title{
Philosophiques
}

\section{Précis de Différence et liberté. Enjeux actuels de l'éducation au pluralisme}

\section{Georges Leroux}

Volume 43, numéro 2, automne 2016

URI : https://id.erudit.org/iderudit/1038215ar

DOI : https://doi.org/10.7202/1038215ar

Aller au sommaire du numéro

Éditeur(s)

Société de philosophie du Québec

ISSN

0316-2923 (imprimé)

1492-1391 (numérique)

Découvrir la revue

Citer ce document

Leroux, G. (2016). Précis de Différence et liberté. Enjeux actuels de l'éducation au pluralisme. Philosophiques, 43(2), 465-469. https://doi.org/10.7202/1038215ar d'utilisation que vous pouvez consulter en ligne.

https://apropos.erudit.org/fr/usagers/politique-dutilisation/ 


\title{
Disputatio
}

\section{Précis de Différence et liberté. Enjeux actuels de l'éducation au pluralisme'}

\author{
GEORGES LEROUX \\ Département de philosophie \\ Université du Québec à Montréal \\ leroux.georges@uqam.ca
}

Dans les sociétés démocratiques, l'éducation au pluralisme est devenue une responsabilité publique dont tous les systèmes d'éducation découvrent à la fois l'urgence et la complexité. En raison de la croissance de la diversité, sur tous les plans où elle est aujourd'hui observée, les gouvernements se sont attelés à la tâche de mettre en place dans les écoles divers modèles: qu'il s'agisse de l'éducation interculturelle, d'éducation à la citoyenneté, de philosophie pour enfants, tous ces modèles ont en commun le souci de donner aux jeunes les instruments pour accéder dans la vie adulte au débat démocratique et au respect de la diversité. Certaines sociétés, comme la France, disposent de traditions républicaines dont les valeurs constituent les objets privilégiés de l'éducation aux normes démocratiques et elles cherchent à en renouveler la transmission; d'autres, comme la société américaine, ne possèdent pas de modèle unitaire auquel se référer, et on y observe un processus très diversifié de recherche curriculaire, rendu nécessaire par la variété des situations éducatives.

La situation du Québec se caractérise par un processus de déconfessionnalisation du système scolaire beaucoup plus lent qu'ailleurs, qu'on peut décrire comme un délestage progressif de l'enseignement confessionnel et comme l'émergence d'un nouveau modèle fondé sur l'éducation morale. Dans le système à options qui a prévalu dans la phase de transition, nous observons en effet que l'option de l'éducation morale correspondait au choix des parents qui n'étaient pas désireux de donner à leurs enfants une éducation religieuse. Ce choix par défaut ne constituait pas une option universalisable, et on ne doit pas s'étonner qu'il ait été abandonné. En 2005, le gouvernement a en effet mis en place le programme Éthique et culture religieuse, dont les principes avaient été énoncés dans le rapport du Groupe de travail sur la place de la religion à l'école, présidé par Jean-Pierre Proulx. Un des résultats les plus nets de ce rapport était de consacrer l'importance du principe de l'égalité au sein de l'école et de fonder cette égalité sur une transmission respectueuse non seulement de la diversité des croyances et de l'in-

1 Montréal, Boréal, 2016.

PHILOSOPHIQUES 43/2 - Automne 2016, p. 465-469 
croyance, mais également de la diversité des conceptions morales de la justice et du bien. C'est à partir de la connaissance de cette diversité que se constitue le pluralisme, qui est indissociablement religieux et moral: le double domaine de l'éthique, conçue comme réflexion sur les principes de l'agir humain, et de la culture religieuse, conçue comme connaissance des systèmes symboliques, apparaît donc comme le résultat de cette longue évolution.

Cette décision illustre le projet de ne pas laisser vacante la place du savoir moral et symbolique de la société dans l'éducation. Ces deux champs possédant leur autonomie historique, on aurait pu décider de les sacrifier au profit de savoirs en apparence plus utiles. On aurait pu également ne retenir que l'éthique, dans une forme ou une autre des modèles éducatifs présents aujourd'hui dans les systèmes d'éducation dans le monde. Le gouvernement a pris la décision de les associer de manière ferme, et d'y joindre, dans une perspective résolument démocratique, une éducation à la compétence du dialogue.

La formation à l'éthique poursuit une compétence nettement ajustée aux exigences du pluralisme: conduire chaque jeune à une réflexion critique et personnelle sur les grands enjeux moraux de notre temps. Il ne s'agit donc plus d'une éducation morale au sens traditionnel, mais d'une éducation à la compétence de discuter et de juger des principes éthiques qui justifient l'action morale. Par contraste, la compétence de la culture religieuse poursuit une finalité très différente, puisque son objet n'est pas l'accès à des principes mais plutôt à un ensemble de connaissances concernant les croyances et l'incroyance, dont la diversité impose une approche non confessionnelle du phénomène religieux. Le fondement demeure celui de l'éducation au pluralisme, chaque jeune étant invité à développer des outils de compréhension en même temps qu'une interprétation personnelle de la religion comme fait social et culturel. Ouvrir la fenêtre sur un monde pluriel, c'est aller à la rencontre de la diversité du savoir moral et symbolique de la société, tenter de la connaître et de la comprendre.

Ce choix d'une approche complexe est-il justifié ? Dans ce livre, prenant appui sur les acquis de la philosophie politique contemporaine, je soutiens que cette approche repose sur un ensemble d'arguments qui éclairent la situation du Québec. Les penseurs auxquels je me réfère appartiennent pour la plupart à la tradition libérale, principalement américaine et britannique. Chaque société cultive un savoir relatif aux enjeux moraux qui la traversent, et nous devons au philosophe Jürgen Habermas d'avoir mis en lumière l'importance de ce savoir comme trame constitutive de la vie sociale. Chaque société recueille également la contribution symbolique des croyances et de l'incroyance à la compréhension de la place de l'individu et de la collectivité dans le monde. Le savoir moral s'exprime ultimement dans les principes juridiques de nos chartes de droits, mais il imprègne également tout ce qui concerne la délibération collective sur les principes éthiques. L'exemple de la justice sociale illustre particulièrement ce double registre: l'évolution de la 
pensée sur les droits demeure en effet tributaire de la réflexion collective sur des problèmes comme ceux de l'inégalité persistante, la discrimination, etc.

L'éducation au pluralisme doit donc inclure l'ensemble des vecteurs du pluralisme dans les sociétés démocratiques, autant le pluralisme moral que le pluralisme religieux. La richesse de ce pluralisme, qui comprend également toutes les positions de l'humanisme séculier qui se retrouvent dans l'un et dans l'autre, constitue une richesse substantielle. L'évolution des démocraties vers un formalisme juridique centré sur les chartes de droits peut avoir pour effet de minimiser, voire d'occulter, l'importance des convictions morales et des croyances qui se trouvent au fondement de l'action humaine. Une éducation à la citoyenneté qui se concentrerait uniquement sur les chartes de droits serait très limitée et priverait les jeunes de la connaissance des normes et des croyances. Dans l'éducation traditionnelle, les principes étaient transmis sous la forme de vertus et de valeurs, que l'enseignement religieux et moral avait charge de communiquer, notamment au moyen d'un répertoire symbolique commun, comme celui de la littérature. Aujourd'hui, les exigences de la laïcité conduisent à un respect critique de la diversité, que cette laïcité a la responsabilité de protéger en fonction de nos principes de liberté. Le pluralisme substantiel devient ainsi un pluralisme normatif, et c'est sur ce concept que peut s'appuyer une éducation au pluralisme en accord avec la situation de notre temps.

De quoi s'agit-il ? Il me semble important de distinguer trois registres très différents qui cohabitent au cœur de cette discussion. Le premier est le registre factuel de la diversité, que nous pourrions qualifier de diversité sociologique. Cette diversité correspond à des faits observables, tels que la multiplicité des appartenances religieuses ou la multiplicité des règles morales qui régissent l'action des individus et des groupes. Dans la philosophie libérale, chez John Rawls par exemple, c'est le respect de cette diversité qui rend nécessaire une réflexion sur les principes de l'équité et le recours au concept du pluralisme. C'est ainsi que nous accédons au second registre, celui du concept philosophique du pluralisme, dont la problématique réside dans le rapport à la vérité et à la justice: toutes les conceptions de la justice et du bien sont-elles équivalentes? Peut-on chercher à les réduire à une conception unifiée? L'État est-il l'unique garant du respect de la diversité? Le problème philosophique du pluralisme réside dans l'ensemble des questions qui résultent du constat de la diversité. Il y a enfin un troisième registre, c'est celui du pluralisme comme valeur ou comme norme, et que nous désignons comme le registre du pluralisme normatif: en quoi une attitude respectueuse du pluralisme peut-elle constituer une norme pour les sociétés démocratiques?

Les justifications de cette position qui sont apportées par la philosophie politique sont très nombreuses, mais on les résumerait bien en disant que toutes ces justifications se fondent sur la qualification normative du pluralisme, autant moral que religieux, pour soutenir l'évolution démocra- 
tique de nos sociétés. C'est seulement en discutant non seulement la différence observable des options mais aussi les problèmes résultant de notre jugement sur le pluralisme que nous pouvons accéder au registre supérieur de la délibération collective: sommes-nous prêts à accepter le pluralisme, et à le considérer comme une richesse plutôt que comme un défaut ou un obstacle? Quelles sont les règles du vivre-ensemble que nous voulons formuler pour respecter les principes de l'égalité et de la liberté? Quel est le rôle de l'éducation dans la connaissance et le partage de ces règles?

À ce programme complexe, deux finalités complémentaires ont été assignées: la reconnaissance de l'autre et la poursuite du bien commun. Ces deux finalités fondamentales sont des finalités sociales, elles sont mises de l'avant en conformité avec la poursuite d'un idéal démocratique, et ce sont elles qui constituent les principes de la philosophie publique de l'éducation qui découle de la nouvelle situation de laïcité scolaire. Il appartient à la réflexion philosophique de les discuter, et notamment de préciser leur convergence avec les principes de l'égalité et de la liberté. Il en va de même pour l'énoncé des droits.

Plusieurs conceptions inégalitaires entrent en conflit avec le principe de l'égalité, et notamment avec la reconnaissance de la place des femmes ou des minorités dans la société. On en dirait autant de conceptions autoritaires, peu respectueuses du principe de liberté. Ces principes sont un acquis de la doctrine des droits, ils sont inscrits dans nos chartes et ne sauraient être l'objet d'accommodements. De manière symétrique, les grandes éthiques philosophiques, par exemple l'utilitarisme, qu'on peut considérer comme la philosophie implicite de notre époque, subissent régulièrement le choc des exigences religieuses relatives à la justice, dans la mesure où leurs acquis rationnels paraissent très pauvres eu égard aux requêtes fondamentales des religions relatives au bien.

Dans cette approche complexe des principes éthiques et des connaissances du fait religieux, le but poursuivi est de comprendre ce qui distingue les croyances des raisonnements proposés par la réflexion éthique et ce qui identifie les religions comme univers de signification construits au sein de communautés toujours particulières et historiques. La question philosophique de l'éducation au pluralisme n'est donc pas identique à celle de l'éducation à la citoyenneté, même si plusieurs convergences peuvent être identifiées. Les jeunes peuvent en effet s'approcher des faits constitutifs de leur environnement social et apprendre à les considérer dans leur singularité. Le choix par chacun de la priorité de la raison ou de la croyance, ou plus exactement la question de l'équilibre pour chacun de la raison et de la croyance apparaît comme le thème cognitif de ce programme: à travers la diversité des conceptions du monde et des représentations auxquelles il aura accès, chaque jeune sera invité à poser cette question pour lui-même. Tel est aujourd'hui l'enjeu du pluralisme. Cela n'exclut aucunement de s'interroger sur les fondements des croyances, mais la perspective doit être compréhen- 
sive et pleinement herméneutique: comprendre les dimensions de l'univers de sens de l'autre, comme on comprend un langage différent du sien, et aller à sa rencontre.

Il y a donc un enjeu épistémologique fondamental dans ce programme, un enjeu relatif à la nature de ce double savoir de l'éthique et de la connaissance culturelle des religions: c'est celui de considérer comme des connaissances de même niveau et de même portée tout ce qui concourt à comprendre les traditions des croyances et des normes au sein de la vie sociale. Ces questions me semblent l'exemple d'une réflexion philosophique sur le pluralisme à laquelle chaque élève devrait être amené au cours de sa formation.

Cette problématique nous met en présence d'un double défi. D'une part, l'éducation au pluralisme comme tâche politique de notre société découle de la nécessité d'une éducation à la citoyenneté qui soit en cohérence avec l'ensemble du système éducatif. On évoque souvent le vivreensemble comme défi contemporain majeur face à la diversité, et je reconnais en effet dans ce défi le principe moteur de l'éducation au pluralisme dans toute sa complexité. Nous sommes appelés, d'autre part, à donner à ce projet un contenu, la construction d'une culture publique commune pour tous les jeunes du Québec. Cette culture est en phase d'érosion, et nous devons y réagir à partir de priorités démocratiques nouvelles, qui ne peuvent uniquement se fonder sur l'humanisme traditionnel.

Toutes ces questions sont au cœur de mon essai, mon but étant de mettre en relief la contribution de l'école à l'évolution vers un pluralisme normatif enrichi et partagé. Le Québec, toujours tenté par une approche utilitariste de l'éducation, ne cesse de l'envisager. L'avènement d'une culture pluraliste, centrée sur des finalités démocratiques et nourrie à plusieurs sources culturelles, me semble une meilleure réponse. La perspective humaniste que je défends ici en propose l'illustration. 